\title{
IMPACT OF LAPAROSCOPIC OVARIAN CYSTECTOMY ON OVARIAN VOLUME, ANTRAL FOLLICLE COUNT (AFC) AND OVARIAN DOPPLER VELOCIMETRY
}

\author{
By
Mohamed Salah Sayed Saadawy, Emad Maarouf Abd El-Latif and Mofeed Fawzy Mohamed

Department of Obstetrics and Gynecology, Faculty of Medicine- Al-Azhar University

*Correspondence author: Mohamed Salah Sayed Saadawy,

E-mail: m.salah_86@yahoo.com

\begin{abstract}
Background: Ovarian cysts are common in reproductive-aged women. Most cysts are normal functional cysts that typically resolve spontaneously and do not require treatment. However, pathologic cysts such as endometriomas and dermoids may require surgical intervention.

Objective: To evaluate the impact of laparoscopic ovarian cystectomy on ovarian reserve regarding ovarian volume, antral follicle count (AFC) and ovarian doppler velocimetry.

Patients and Methods: A prospective observational study was conducted at 15 May Specializes Hospital on 80 women, less than 35 years old, with single unilateral ovarian cyst (endometriotic or non-endometriotic) ovarian cysts. Presence of ovarian cyst was diagnosed by preoperative transvaginal ultrasound (TVUS) scan which was confirmed at laparoscopy. All laparoscopic ovarian cystectomies were performed under general anesthesia.
\end{abstract}

Results: There was a statistically significant decrease in ovarian volume (OV) volume and AFC in operated side than contralateral side while no statistically significant difference found between them regarding resistive index (RI).

Conclusions Laparoscopic ovarian cystectomies were risk factors for decreased ovarian reserve and may impair the future fertility.

Keywords: Antral follicle count, Transvaginal ultrasound, laparoscopic ovarian cystectomies.

\section{INTRODUCTION}

Ovarian cyst is commonly discovered in women of reproductive age generally as well as in those undergoing work-ups for infertility. The differential diagnosis of an ovarian cyst discovered in women of reproductive age is broad, including physiologic or functional cysts, ectopic pregnancy, inflammatory etiologies such as a tuboovarian abscess, endometrioma or benign and malignant ovarian neoplasms. The most common benign ovarian masses are serous and mucinous cystadenoma, endometrioma and mature cystic teratoma. Many ovarian masses in the premenopausal woman can be managed conservatively. Functional or simple ovarian cysts (thin-walled cysts without internal structures) which are less than $50 \mathrm{~mm}$ maximum diameter usually 
resolve over 3 cycles without need for intervention (Practice Committee of the American Society, 2012).

Endometriosis is an enigmatic disease characterized by development of the endometrial tissue outside of the uterus. The most common location of endometriosis is the ovary, occurring in $17-44 \%$ of patients affected by endometriosis. Endometrioma is the formation of ovarian cyst lined by the endometrial glands and stroma (Nelson, 2013).

The concept of ovarian reserve views reproductive potential as a function and quality of number of the remaining oocytes. Decreased or diminished ovarian reserve (DOR) describes women of reproductive age having regular menses whose response to ovarian stimulation or fecundity is reduced compared with women of comparable age. Decreased ovarian reserve is distinct from menopause or premature ovarian failure (Cooper et al., 2012).

Although ovarian reserve tests have been applied widely, debate continues over the ability of tests currently in use to predict three related outcomes: oocyte quality, oocyte quantity and fecundity. The goal of ovarian reserve testing is to add more prognostic information to the counseling and planning process so as to help couples choose among treatment options (Rustamov et al., 2012).

Ovarian reserve tests include both biochemical tests and ultrasound imaging of the ovaries. Biochemical tests of ovarian reserve can be divided further into basal measurements including measurement of follicle-stimulating hormone, estradiol, inhibin $\mathrm{B}$ and antimullerian hormone and provocative tests such as the clomiphene citrate challenge test (CCCT). Ultrasonographic measures of ovarian reserve include antral follicle count (AFC) and ovarian volume. Ovarian doppler velocimetry has not been extensively used to evaluate post-surgical ovarian damage. Being related to microvascular bed: this parameter was higher in the operated than in the nonoperated ovary independently on the histological type of the removed cysts (Cagnacci et al., 2016).

Antral follicle count (AFC) has been demonstrated to be a reliable marker of ovarian reserve, since it correlates significantly with the age-related follicle count decline, and with ovarian response to in-vitro fertilization (IVF) stimulation cycles (Shah et al., 2014).

The most common side effect of ovarian cystectomy is damage to healthy ovarian tissue therefore; application of new techniques with lower adverse effects on ovarian reserve is a current concern (Asgari et al., 2015).

Compared with traditional surgery by laparotomy, laparoscopic approach has been shown to be feasible in the vast majority of cases and when associated with a shorter hospital stay, faster recovery, less pain and lower costs (Ding et al., 2015).

Laparoscopic cystectomy is the gold standard for managing endometriomas. Laparoscopic cystectomy often leads to inadvertent removal of an amount of the adjacent ovarian cortex and serious bleeding at the ovarian hilus requiring extensive application of bipolar electrocoagulation and hence, adverse changes in ovarian blood supply, as well 
as a functional loss in the ovarian reserve (Mircea et al., 2016). Mini-laparotomy may be considered for occasional very large cysts of benign appearance. On rare occasions the laparoscopic approach may be specifically contraindicated in an individual patient (Hayasaka et al., 2010).

The aim of the present work was to evaluate the impact of laparoscopic ovarian cystectomy on ovarian reserve regarding ovarian volume, antral follicle count (AFC) and ovarian doppler velocimetry.

\section{PATIENTS AND METHODS}

The current study was a prospective observational study conducted at 15 Mayo specialized Hospital, 80 female who have been subjected to a laparoscopic stripping of a single ovarian cyst on Maternity Hospital, Faculty of Medicine, Al-Azhar University. The presence of unilateral cyst was diagnosed at the preoperative transvaginal ultrasound (TVUS) scan and confirmed at laparoscopy from June 2018 to December 2019.

In the current study, we excluded women with more than one ovarian cyst either unilateral or bilateral, previous ovarian surgery, suspected ovarian malignancy and pregnancy.

An approval of the study was obtained from Al- Azhar University academic and ethical committee. Every patient signed an informed written consent for acceptance of the operation.

\section{The recruited patients were subjected} the following:

i. Complete history taking and pelvic examination. ii. TVUS: Single benign monolateral ovarian cyst was diagnosed by Preoperative transvaginal ultrasound (TVUS) scan which was confirmed at laparoscopy. Diameter of the cyst was recorded at time of the preoperative TVUS.

iii. Laparoscopy: All laparoscopic ovarian cystectomies was performed under general anaethesia by the same gynecologic surgeon using with a standardized technique.

iv. TVUS: Eligible women were asked to repeat a TVUS after two months of laparoscopy. All the TVUS was performed by the same operator at day 3-6 of menstruation.

The following parameters were evaluated:

i. Ovarian volume: volume of both operated and contralateral ovary was evaluated by the prolate ellipsoid (PE) method (LD x APD xTD x 0.5233).

ii. AFC: All echofree structures in the operated and contralateral ovary with a mean diameter of $2-10 \mathrm{~mm}$ were counted as an antral follicle.

iii. Ovarian Doppler: RI of ovarian artery of operated and contralateral ovary. Ovarian arteries are displayed in the infundibulo- pelvic ligament at the infero-lateral side of the ovaries.

\section{Statistical method:}

The collected data were coded, tabulated, and statistically analyzed using SPSS program (Statistical Package for the Social Sciences) software version 25. Descriptive statistics were done for parametric quantitative data by mean and Standard deviation, median and 
interquartile range (IQR), while they were done for categorical data by number and percentage. Distribution of the data was done by Kolomogorov Smirnov test. Analyses were done for parametric quantitative data between the two groups using independent samples $\mathrm{T}$ test. Or Mann-whitneg test The level of significance was taken at $(\mathrm{P}$ value $<0.05)$.

\section{RESULTS}

This study was conducted on 80 women recruited during the period from $6 / 2018$ to $12 / 2019$. Their ages ranged from 18-34 years with Mean \pm SD of 24.28 \pm 4.30. Their parity ranged from $0-4$ with median and IQR of 1 0-1 (Table 1).

Table (1): Age, parity, anthropometric measures, cyst diameter and type among of the studied cases

\begin{tabular}{|c|c|c|}
\hline \multicolumn{2}{|l|}{ Parameters } & No. $=80$ \\
\hline \multirow{2}{*}{ Age (years) } & Mean \pm SD & $24.28 \pm 4.30$ \\
\hline & Range & $18-34$ \\
\hline \multirow{2}{*}{ Parity (No) } & Median(IQR) & $1(0-1)$ \\
\hline & Range & $0-4$ \\
\hline \multirow{2}{*}{ Height $(\mathrm{cm})$} & Mean \pm SD & $163.04 \pm 9.87$ \\
\hline & Range & $145-188$ \\
\hline \multirow{2}{*}{ Weight $(\mathrm{kg})$} & Mean \pm SD & $73.55 \pm 16.02$ \\
\hline & Range & $45-111$ \\
\hline \multirow{2}{*}{ BMI $\left(\mathrm{kg} / \mathrm{m}^{2}\right)$} & Mean \pm SD & $27.41 \pm 3.92$ \\
\hline & Range & $19.22-35.11$ \\
\hline \multirow{2}{*}{ Cyst diameter } & Mean \pm SD & $7.40 \pm 2.27$ \\
\hline & Range & $3.9-14$ \\
\hline \multirow{2}{*}{ Cyst type } & Non-endometrial & $57(71.3 \%)$ \\
\hline & Endometrial & $23(28.8 \%)$ \\
\hline
\end{tabular}

There was a statistically significant decrease in OV volume and AFC in operated side than contralateral with $\mathrm{p}$ value $<0.001$ and $<0.001$ respectively while no statistically significant difference found between them regarding RI with $\mathrm{p}$ value $=0.204$ (Table 2$)$.

Table (2): Comparison between operated side and contralateral side regarding OV volume, AFC and RI

\begin{tabular}{|c|c|c|c|c|}
\hline \multirow{2}{*}{\multicolumn{2}{|c|}{ Parameters Side }} & Operated & Contra lateral & \multirow{2}{*}{ P-value } \\
\hline & & No. $=80$ & No. $=80$ & \\
\hline \multirow{2}{*}{ OV volume } & Mean \pm SD & $5.26 \pm 1.87$ & $7.33 \pm 2.75$ & \multirow{2}{*}{$<0.001$} \\
\hline & Range & $2.5-11$ & $3-15$ & \\
\hline \multirow{2}{*}{ AFC } & Mean \pm SD & $3.16 \pm 1.85$ & $6.89 \pm 2.77$ & \multirow{2}{*}{$<0.001$} \\
\hline & Range & $1-8$ & $2-12$ & \\
\hline \multirow{2}{*}{ RI } & Mean \pm SD & $0.80 \pm 0.14$ & $0.73 \pm 0.15$ & \multirow{2}{*}{$<0.002$} \\
\hline & Range & $0.4-1.1$ & $0.48-1$ & \\
\hline
\end{tabular}


There was no a statistically significant correlation found between $\mathrm{OV}$ volume and RI and the other studied parameters. The table also shows that there was statistically significant positive correlation found between AFC with weight and BMI of the studied cases (Table 3 ).

Table (3): Correlation of $\mathrm{OV}$ volume, AFC and RI of the operated side with demographic and anthropometric measures of the studied patients

\begin{tabular}{|l|c|c|c|c|c|c|}
\hline \multirow{2}{*}{ Operated } & \multicolumn{2}{c|}{ OV volume } & \multicolumn{2}{c|}{ AFC } & \multicolumn{2}{c|}{ RI } \\
\cline { 2 - 7 } Parameters & r & P-value & r & P-value & r & P-value \\
\hline Age & -0.017 & 0.880 & -0.101 & 0.371 & 0.110 & 0.331 \\
\hline Height & -0.101 & 0.375 & 0.017 & 0.884 & -0.011 & 0.924 \\
\hline Weight & 0.022 & 0.845 & $0.261^{*}$ & 0.019 & -0.069 & 0.540 \\
\hline BMI & 0.082 & 0.470 & $0.401^{* *}$ & 0.000 & -0.080 & 0.479 \\
\hline Parity & -0.056 & 0.622 & 0.111 & 0.327 & 0.047 & 0.681 \\
\hline Cyst diameter & -0.151 & 0.181 & -0.021 & 0.855 & 0.110 & 0.329 \\
\hline
\end{tabular}

There was no a statistically significant other studied parameters in operated and relation found between cyst type and the contralateral sides (Table 4).

Table (4): Relation of cyst type with the studied parameters in operated and contralateral sides

\begin{tabular}{|c|c|c|c|c|}
\hline \multirow{2}{*}{\multicolumn{2}{|c|}{ Parameters $\quad$ Cyst type }} & Non-endometrial & Endometrial & \multirow{2}{*}{ P-value } \\
\hline & & No. $=80$ & No. $=80$ & \\
\hline \multicolumn{5}{|l|}{ Operated } \\
\hline \multirow{2}{*}{ OV volume } & Mean \pm SD & $5.2 \pm 1.81$ & $5.4 \pm 2.07$ & \multirow{2}{*}{0.678} \\
\hline & Range & $3-11$ & $2.5-9.5$ & \\
\hline \multirow{2}{*}{ AFC } & Mean \pm SD & $3.14 \pm 1.81$ & $3.22 \pm 2$ & \multirow{2}{*}{0.868} \\
\hline & Range & $1-8$ & $1-8$ & \\
\hline \multirow{2}{*}{ RI } & Mean \pm SD & $0.79 \pm 0.15$ & $0.81 \pm 0.13$ & \multirow{2}{*}{0.367} \\
\hline & Range & $0.4-1.1$ & $0.6-1.1$ & \\
\hline \multicolumn{5}{|c|}{ Contra lateral } \\
\hline \multirow{2}{*}{ OV volume } & Mean \pm SD & $7.28 \pm 2.76$ & $7.46 \pm 2.76$ & \multirow{2}{*}{0.793} \\
\hline & Range & $4-15$ & $3-13$ & \\
\hline \multirow{2}{*}{$\mathrm{AFC}$} & Mean \pm SD & $7.02 \pm 2.87$ & $6.57 \pm 2.56$ & \multirow{2}{*}{0.513} \\
\hline & Range & $2-12$ & $3-11$ & \\
\hline \multirow{2}{*}{ RI } & Mean \pm SD & $0.73 \pm 0.15$ & $0.73 \pm 0.16$ & \multirow{2}{*}{1} \\
\hline & Range & $0.49-1$ & $0.48-1$ & \\
\hline
\end{tabular}

\section{DISCUSSION}

Laparoscopic surgery is currently considered the treatment of choice in women with benign ovarian cysts and has gained increasing acceptance among gynecologic surgeons (Hayasaka et al., 2010). Serum concentrations of folliclestimulating hormone, luteinizing hormone, estradiol, and inhibin, as well as ovarian volume and ovarian antral follicle counts, may be useful, but are not necessarily considered highly predictive values. Therefore, using those hormonal tests may be not informative enough to assess postsurgical ovarian reserve precisely (Guleria et al., 2018).

Comparison between the previously operated ovary with the contralateral 
intact ovary reflected the influence of the surgery more accurately. This technique has been used for treating ovarian endometriotic cysts by five independent researchers, and for treating ovarian non endometriotic cysts by another researcher (Somigliana et al., 2012).

The ovarian follicular heritage undergoes a slow decline during the reproductive life. There is a slew of more or less validated markers of ovarian reserve, that in general can be separated in serum (follicle stimulating hormone (FSH), estradiol (E2), inhibin B and anti$\mathrm{Mu}$ "llerian hormone (AMH)) and ultrasound-determined (ovarian volume, antral follicle count (AFC)). Some exogenous insults, like gonadal surgery, can speed up the process of ovarian follicle loss and lead to early menopause (Cagnacci et al., 2016).

Surgery for endometriomas has been associated with a risk of reduced ovarian reserve, documented with a decrease of several ovarian markers like AFC, AMH and volume. Furthermore, it was demonstrated that ovarian color Doppler velocimetry changed after endometriomas removal, with an increase of the resistance index (RI) (Muzii et al., 2014).

This study was selected to evaluate the impact of laparoscopic ovarian cystectomy on the ovarian volume, antral follicle count (AFC) and ovarian doppler velocimetry.

The main results of this study were: Their age ranged from 18-34 years with mean \pm SD of $24.28 \pm 4.30$ and their parity ranged from $0-4$ with median and IQR of 1 (0-1).
Our results were in agreement with study of Salem et al. (2017) as they found that the mean of age of their participants was $24.5 \pm 4.5$ years. Our results are supported by study of Asgari et al. (2015) as they reported that the mean age \pm standard deviation (SD) was $29.72 \pm 5.93$ of the studied patients.

The present study showed that the mean of BMI of our patients was $27.41 \pm$ 3.92. Our results were in line with study of Salem et al. (2017) as they reported that the mean of BMI of their studied patients was $26.24 \pm 5.2$.

In the study in our hands, the mean of cyst diameter was $7.40 \pm 2.27$ and $71.3 \%$ had non -endometrial cysts and $28.8 \%$ of them had endometrial cysts.

Our results were supported by study of Cagnacci et al. (2016) as they reported that in $60.6 \%$, a non-endometriotic cyst was removed (16 teratomas, 12 simple cysts, 10 serous cysto-adenomas, 5 mucinous cysto-adenomas). The rest of the cases were endometriomas (28/71, $39.4 \%$ ).

According to Ding et al. (2015) seventy patients underwent laparoscopic ovarian cystectomy, with bilateral endometrioma $(\mathrm{n}=21)$, unilateral endometrioma $(\mathrm{n}=29)$, and unilateral other benign ovarian cyst $(n=20)$ were included in the study.

However, decreased ovarian reserve which is due to surgical removal of a part of the healthy ovarian tissue together with the endometrioma wall is considered as a major concern after surgery leading to the risk of premature ovarian failure.

The present study showed that there was a statistically significant decrease in 
$\mathrm{OV}$ volume and AFC in operated side than contralateral, while no statistically significant difference found between them regarding ovarian Doppler RI.

Our results were supported by the study of Cagnacci et al. (2016) who reported that both in endometriotic and non-endometriotic cysts the mean volume and AFC of the operated ovary was significantly lower than that of the contralateral non-operated ovary, while RI was significantly higher.

Asgari et al. (2015) revealed that treatment type was negatively correlated with FSH level, so that surgery with the suturing technique had lower FSH concentrations with higher ovarian reserve as compared with patients in bipolar coagulation group.

Furthermore, two other studies conducted by Zaitoun et al. (2013) and Mohamed et al. (2011) compared ovarian reserve between laparoscopic ovarian cystectomy and open laparotomy with hemostatic suturing and both indicated a significant decrease in ovarian reserve in bipolar group as compared to open suture group.

Other techniques have been also proposed for protecting ovarian reserve in women undergoing ovarian surgery. Song et al. (2014) suggested use of hemostatic sealant as a safe technique with fewer side effects on ovarian reserve as compared with bipolar coagulation.

However, Ding et al. (2015) reported that there was no detectable difference on ovarian reserve marker levels between 4 groups and from baseline values at 6 and 12 months after laparoscopic ovarian cystectomy of endometrioma, although these levels significantly declined in the first month postoperatively.

Findings of our results are in contrast with study of Asgari et al. (2015) as they reported that age was a significant predictor of $\mathrm{AMH}$. Ovarian reserve is marked as the size and quantity of the remaining ovarian follicular pool at any given time. AMH level has been considered as a reliable and useful marker of ovarian reserve than the levels of inhibin B, estradiol (E2), folliclestimulating hormone (FSH) and luteinizing hormone (LH) on cycle day.

Furthermore, Hayasaka et al. (2010) demonstrated that age at the time of surgery, the diameter of the excised cysts, age at the time of ICSI, basal FSH, bodymass (BMI), duration of stimulation, total amount of gonadotropin, and peak estradiol at the time of ICSI were not significantly different between groups (patients with ovarian endometriotic mono-lateral cyst, patients with ovarian non-endometriotic mono-lateral cysts.

Cagnacci et al. (2016) reported that mean diameter of the removed cysts, significantly smaller for endometriotic than non-endometriotic cysts.

Our results were supported by study of Cagnacci et al. (2016) as they had studied the impact on ovarian reserve of the removal of an endometriotic and nonendometriotic cyst, using ultrasounddetermined markers like ovarian volume and AFC. The results showed that the removal of any ovarian cyst reduces follicular ovarian reserve in a similar way.

Although in the case of endometriomas, ovarian reserve may be affected by the presence of the disease 
itself. 22 Studies evaluating ovarian reserve by Muzii et al. (2014), volume or even AMH15 reported, almost consistently, that ovarian reserve is reduced by the surgical removal of an endometriotic cyst.

In another study conducted by Takashima et al. (2013), patients undergoing laparoscopic excision of a unilateral ovarian endometrioma showed no significant differences in the serum levels of AMH and FSH were found at 3 months after surgery compared with preoperative levels. Their findings may be due to smaller sample size and use of diluted vasopressin into the cyst wall which reduced both oozing and use of hemostatic methods.

Sugita et al. (2013) also evaluated the serum AMH levels at 1 month and 1 year after cystectomy for endometrioma. Although some patients showed higher AMH levels at 1 year after surgery than 1 month after surgery, they suggested ovarian reserve decreased just after surgery, but recovered within 1 year.

The results of the study of Hayasaka et al. (2010) suggest that ovarian reserve is decreased more significantly after excision of endometriotic cysts than after surgery for non-endometriotic cysts, possibly not only because of the surgical procedure but also because of the presence of endometriosis in the pelvic cavity.

Data on non-endometriotic ovarian cysts obtained with AFC reported either a reduction or no effect of cyst removal, in spite of a reduced ovarian volume. Biochemical indexes of ovarian reserve such as AMH declined following the removal of non-endometriotic ovarian cysts (Kwon et al., 2014).

\section{CONCLUSION}

Ovarian cystectomies are risk factors for decreased ovarian reserve and may impair the future fertility.

\section{REFERENCES}

1. Asgari Z, Rouholamin S, Hosseini R, Sepidarkish $M$ and Hafizi L. (2015): Comparing ovarian reserve after laparoscopic excision of endometriotic cysts and hemostasis achieved either by bipolar coagulation or suturing: a randomized clinical trial, Arch Gynecol Obstet., 15(5): 48-59.

2. Cagnacci A, Bellafronte M, Xholli A, Palma $F$ and Maddalena M. (2016): Impact of laparoscopic cystectomy of endometriotic and non-endometriotic cysts on ovarian volume, antral follicle count (AFC) and ovarian doppler velocimetry, Gynecol Endocrinol., 1: 11:18.

3. Cooper AR, Baker VL, Sterling EW, Ryan ME and Woodruff TK. (2013): The time is now for a new approach to primary ovarian insufficiency. Fertil Steril., 95:1890-7.

4. Ding $Y$, Yuan $Y$, Ding $J$, Chen $Y$ and Zhang X. (2015): Comprehensive assessment of the impact of laparoscopic ovarian cystectomy on ovarian reserve. Journal of Minimally Invasive Surgery, 1(2):7-11.

5. Guleria S, Jensen A and Kjær SK. (2018): Risk of borderline ovarian tumors among women with benign ovarian tumors: A cohort study. Gynecologic Oncology, 148(1): 86-90.

6. Hayasaka S, Arai M, Ugajin T, Nabeshima $H$ and Utunomiya H. (2010): Comparing Ovarian Reserves After Laparoscopic Excision of Ovarian Endometriotic Cysts and Ovarian Nonendometriotic Cysts. Journal of Gynecologic Surgery, 26(4): 237-241.

7. Kwon SK, Kim SH, Yun SC, Kim DY and Chae HD. (2014): Decline of serum antimüllerian hormone levels after laparoscopic ovarian cystectomy in endometrioma and other benign cysts: a prospective cohort study. Fertility and Sterility, 101(2): 435-441. 
8. Mircea O, Bartha E, Mihai Gheorghe M, Irimia $T$ and Radu V. (2016): Ovarian Damage after Laparoscopic Cystectomy for Endometrioma. Chirurgia., 111: 54-57.

9. Mohamed ML, Nouh AA, El-Behery MM and Mansour SA. (2011): Effect on ovarian reserve of laparoscopic bipolar electrocoagulation versus laparotomic hemostatic sutures during unilateral ovarian cystectomy. International Journal of Gynecology \& Obstetrics, 114(1): 69-72.

10. Muzii L, Di Tucci C, Di Feliciantonio M and Marchetti C. (2014): The effect of surgery for endometrioma on ovarian reserve evaluated by antral follicle count: a systematic review and meta-analysis. Human Reproduction, 29(10): 2190-2198.

11. Nelson SM. (2013): Biomarkers of ovarian response current and future applications. Fertil Steril., 99:963-969.

12. Practice Committee of the American Society for Reproductive Medicine (2012): Testing and interpreting measures of ovarian reserve: a committee opinion. Fertil Steril., 98:1407-1415

13. Rustamov O, Smith A, Roberts SA, Yates AP and Fitzgerald C. (2012): Anti-Mullerian hormone: poor assay reproducibility in a large cohort of subjects suggests sample instability. Hum Reprod., 27:3085-91

14. Salem MN, Ahmed SR, Abbas AM and Salem AN. (2017): Short term effects of laparoscopic ovarian drilling in clomiphene citrate resistant patients with polycystic ovary syndrome. Middle East Fertility Society Journal, 22(4): 290-294.

15. Shah DK, Mejia RB and Lebovic D. (2014): Effect of surgery for endometrioma on ovarian function. J Minim Invasive Gynecol., 21:203209

16. Somigliana $\mathbf{E}$, Berlanda $\mathbf{N}$ and Benaglia $\mathbf{L}$. (2012): Surgical excision of endometriomas and ovarian reserve: a systematic review on serum antimu“llerian hormone level modifications. Fertil Steril., 98: 1531-8.

17. Song T, Lee S-H and Kim WY. (2014): Additional benefit of hemostatic sealant in preservation of ovarian reserve during laparoscopic ovarian cystectomy: a multicenter, randomized controlled trial. Hum Reprod., 29(8):1659-1665.

18. Sugita A, Iwase A, Goto $M$, Nakahara $T$ and Nakamura T. (2013): One-year followup of serum antimüllerian hormone levels in patients with cystectomy: are different sequential changes due to different mechanisms causing damage to the ovarian reserve? Fertility and Sterility, 100(2): 516522.

19. Takashima A, Takeshita N, Otaka $K$ and Kinoshita T. (2013): Effects of bipolar electrocoagulation versus suture after laparoscopic excision of ovarian endometrioma on the ovarian reserve and outcome of in vitro fertilization. J Obstet Gynaecol Res., 39(7):1246-1252.

20. Toner JP and Seifer DB. (2013): Why we may abandon basal folliclestimulating hormone testing: a sea change in determining ovarian reserve using antimu"llerian hormone. Fertil Steril., 99(7):1825-1830.

21. Zaitoun MM, Zaitoun MM and El Behery MM. (2013): Comparing long term impact on ovarian reserve between laparoscopic ovarian cystectomy and open laprotomy for ovarian endometrioma. J Ovarian Res., 6:76-73. 


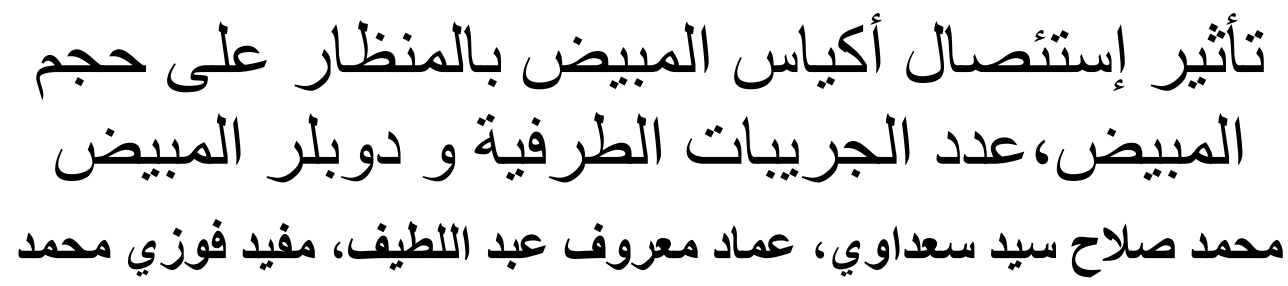

قسم أمراض النساء والتوليد، كلية الطب، جامعة الازهر

E-mail: m.salah_86@yahoo.com

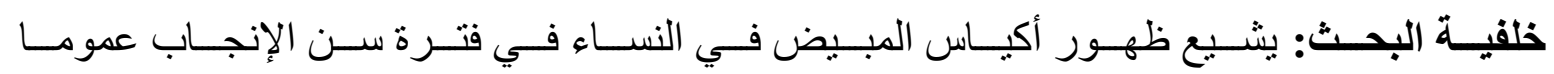

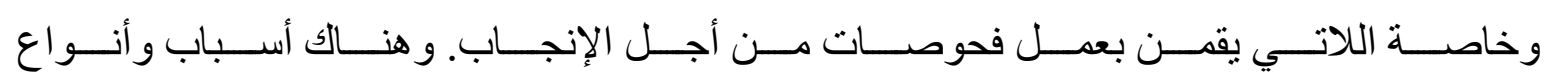

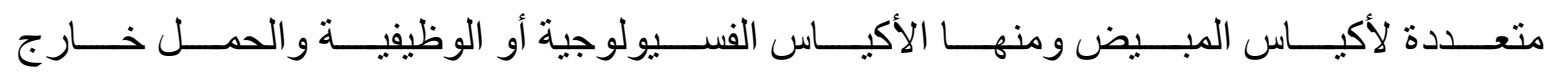

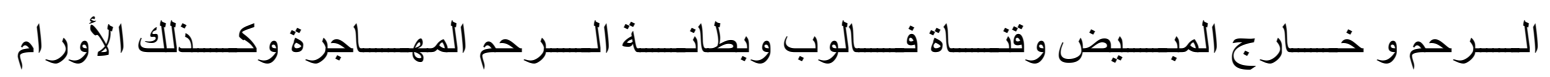

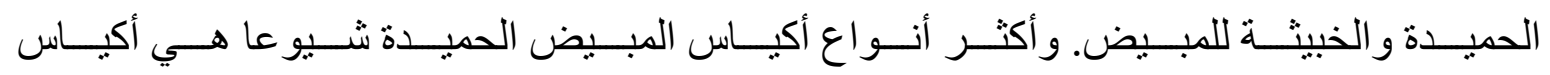
مصلية وميوسينية و أكياس بطانة الرحم المهاجرة وأورام مسخية كيسية ناضجة.

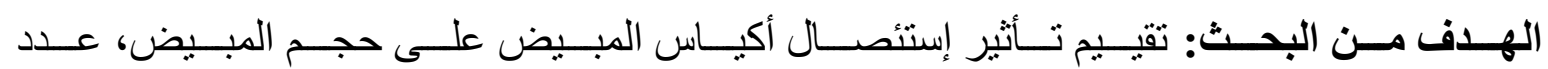
الجريبات الطرفية وكذلك دوبلر المبيض. فئ.

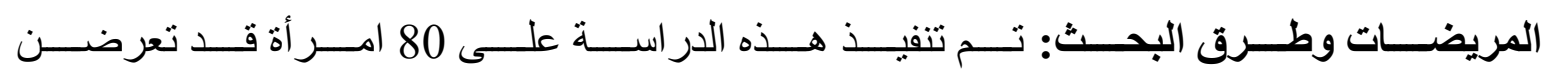

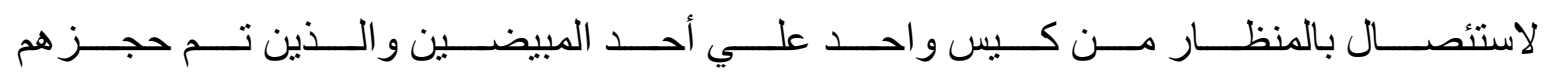

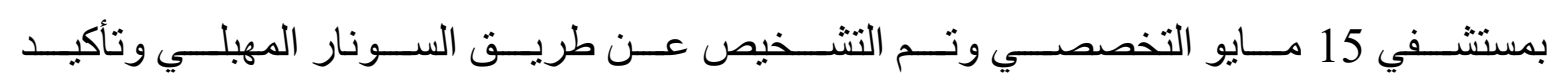
التشخيص عن طريق منظار البطن.

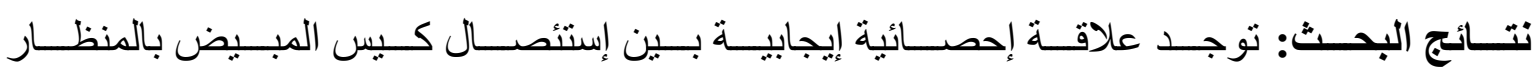
وضعف مخزون المبيض بعد الجر احة.

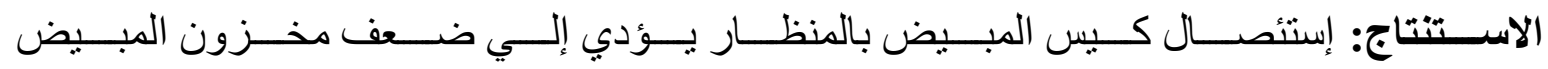

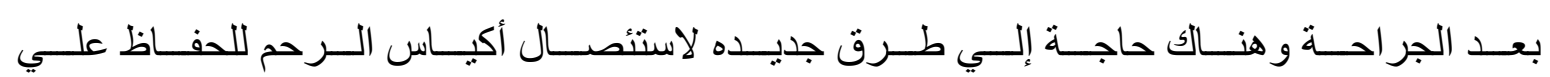
مخزون المبيض وخاصة في حالات تأخر الإنجاب.

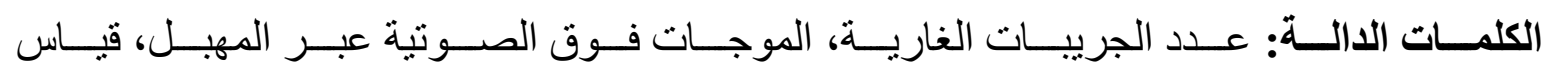
سرعة دوبلر المبيض، إستئصال كيس المبيض بالمنظار. 